\title{
Terminologia Anatomica y Terminologia Histologica. Un Lugar de Encuentro entre los Morfólogos
}

\author{
Terminologia Anatomica and Terminologia Histologica. \\ A Meeting Point between Morphologists
}

\author{
Bélgica Vásquez \& Mariano del Sol ${ }^{* *, * * *, * * * *}$
}

\begin{abstract}
VÁSQUEZ, B. \& DEL SOL, M. Terminologia Anatomica y Terminologia Histologica. Un lugar de encuentro entre los morfólogos. Int. J. Morphol., 34(4):1585-1590, 2015.

RESUMEN: La correcta utilización de las terminologías morfológicas es importante en la comunicación científica. Estas terminologías deben ser claras y precisas y además, concordantes entre ellas. La necesidad de unificar criterios se ha visto reflejada en la realización de los Simposios Ibero-Latinoamericano de Terminología (SILAT), iniciados en el año 2009 por la Asociación Panamericana de Anatomía. Es prioritario unificar y actualizar los términos morfológicos y además, proponer soluciones y plantear desafíos para mejorar las diferentes terminologías. El objetivo del trabajo fue realizar una revisión de algunos términos latinos comunes a Terminologia Anatomica (TA) y Terminologia Histologica (TH), para a manera de ejemplos, comparar e identificar algunos términos que presentan discrepancia y, por tanto, generan confusión para quienes los utilizan. A pesar que ambas terminologías presentan para su confección las mismas consideraciones, tienen algunas diferencias en cuanto a organización, denominación de términos y utilizan más de un término para una misma estructura e incluso, una misma estructura presenta nombres distintos entre una terminología y otra. Numerosos términos están escritos en plural en la $T A$ y singular en la $T H$. De acuerdo a lo anterior, algunas de las mejoras a esta problemática la propone el SILAT, ofreciendo oportunidades para revisar y discutir las distintas terminologías y así evidenciar la necesidad de modificar y armonizar los términos que presentan discrepancias. En resumen, pretendemos que con estos antecedentes iniciales puedan entregarse argumentos que permitan seguir unificando criterios y que ellos puedan ser considerados por los expertos que conforman el Programa Federativo Internacional de Terminología Anatómica y, como bien se señala, permitir el establecimiento de diálogo con los miembros de la Federación Internacional de Asociaciones de Anatomistas e ir mejorando la comunicación científica entre los diferentes actores de las ciencias morfológicas.
\end{abstract}

PALABRAS CLAVE: Terminologías morfológicas; Terminologia Anatomica; Terminologia Histologica.

\section{INTRODUCCIÓN}

La comunicación en las ciencias de la salud requiere de una terminología clara y precisa. En acuerdo con esta necesidad, los Simposios Ibero-Latinoamericanos de Terminología (SILAT), iniciados en el año 2009 por la Asociación Panamericana de Anatomía (APA), tienen por misión divulgar la terminología morfológica internacional, para que las instituciones educativas de medicina y de otras áreas de la salud, de hablas hispana y portuguesa, la empleen cotidianamente (Losardo et al., 2010).

Sin embargo, estos esfuerzos son insuficientes ya que no sólo se debe promover su uso, sino que también es necesario trabajar en conjunto entre los morfólogos para resolver algunas problemáticas que se originan debido, funda- mentalmente, a que diversos términos son utilizados para denominar a una misma estructura. Así, es prioritario unificar y actualizar los términos morfológicos para evitar confusiones, facilitando la enseñanza-aprendizaje (Cruz et al., 2010). Por tal motivo, el SILAT también se preocupa de unificar, especialmente en Latinoamérica las denominaciones de las estructuras morfológicas y así lograr un lenguaje científico común (Losardo et al., 2015). Junto con esto, investigadores de las ciencias morfológicas de distintas nacionalidades comprometidos con la temática, aúnan esfuerzos para exponer problemáticas en el ámbito terminológico, proponer soluciones y plantear desafíos con el fin de mejorar las diferentes terminologías internacionales (Anatómica, Histológica y Embriológica) (Duque Parra et al., 2012;

* Universidad de Tarapacá, Arica, Chile.

** Doctorado en Ciencias Morfológicas, Universidad de La Frontera, Temuco, Chile.

**** Centro de Estudios Morfológicos y Quirúrgicos (CEMyQ), Universidad de La Frontera, Temuco, Chile.

***** Centro de Investigación en Ciencias Biomédicas, Universidad Autónoma de Chile, Temuco, Chile. 
Mejías, 2012; Duque \& Ríos, 2013; del Sol \& Vásquez, 2014; Fuentes et al., 2014; Vásquez \& del Sol, 2014; Roa \& del Sol, 2015).

Este hecho cobra importancia cuando investigaciones recientes ponen en evidencia que el conocimiento de la anatomía en los estudiantes no se obtiene bajo los propósitos de la Terminologia Anatomica (TA), debido a que existe mayor reconocimiento de conceptos médicos básico-clínicos de anatomía que utilizan epónimos dado que el uso de la $T A$ no es reforzada durante estos ciclos del estudiante (Parra et al., 2015).

Recientemente, se ha informado que gracias al patrocinio de las Bibliotecas de la Universidad de Dalhousie (Halifax, Canadá), el Programa Federativo Internacional de Terminología Anatómica (FIPAT) está en condiciones de abrir un segundo sitio Web en <FIPAT.library.dal.ca〉, que en primer lugar, facilitará las actividades de FIPAT y sus Grupos de trabajo (por ejemplo, permite procesar de manera eficiente las respuestas a la consulta TermDropBox actual sobre TA, Terminologia Histologica (TH) y Terminologia Embryologica (TE). En segundo lugar el FIPAT permitirá que se establezca diálogo con los miembros de la Federación Internacional de Asociaciones de Anatomistas (IFAA). De esta manera, en el sitio se podrá acceder a TA (1988), TH (2008) y TE (2013) patrocinado y organizado por la Universidad de Fribourg, Suiza (www.unifr.ch/ifaa). El nuevo sitio web FIPAT en Dalhousie, al igual que el sitio actual en Friburgo, contará con tres zonas: pública, privada y restringida. La zona privada de la web ya está abierta para facilitar el examen del proyecto de revisión de $T A, T H$ y $T E$. En la zona pública se podrán visualizar las terminologías revisadas. Se informó además que, la zona pública FIPAT será el sitio para las próximas versiones validadas de las terminologías IFAA, tan pronto como se hayan completado. La publicación de las terminologías incluirá índices jerárquicos y alfabéticos similares a los de TA de 1998, actualmente en el sitio Friburgo.

La TA es la base de la terminología médica y es importante que los médicos y científicos de todo el mundo utilicen el mismo nombre para cada estructura. Así, el objetivo del presente trabajo fue realizar una revisión de algunos términos latinos comunes a $T A$ y $T H$, con la finalidad de comparar e identificar aquellos que presentan discrepancia y, por tanto, generan confusión. Junto con lo anterior, se espera entregar más antecedentes que permitan unificar criterios que sean $r$ considerados por los expertos que conforman el FIPAT.

\section{Construcción y organización de Terminologia Anatomica e Histologica.}

La TA es la creación conjunta del Comité Federativo de Terminología Anatómica (FCAT) y las 56 asociaciones miembros de la IFAA. Esta iniciativa surgió en la Asamblea General desarrollada durante el XIII Congreso Internacional de Anatomía realizado en Rio de Janeiro, Brasil, en agosto de 1989. Se señala que éste es el único organismo internacional que representa a todos los aspectos de la anatomía y de las asociaciones anatómicas. Posteriormente, en 2006, el FCAT pasa a denominarse Comité Federativo Internacional en Terminología Anatómica (FICAT) cuyo objetivo fue "Presentar la terminología oficial de las Ciencias Anatómicas, previa consulta a todos los miembros de la IFAA, garantizando así una contribución democrática a la terminología". La TA que se encuentra redactada en latín y cuya última actualización se publicó en 1998 y reimpreso en 2011, construyéndose en base a cuatro principios básicos (Latarjet \& Ruiz, 2004):

1. Los nombres de las estructuras deben tener un valor informativo.

2. Se suprime los epónimos debido a que los nombres propios varían entre países.

3. Se suprimen los homónimos para evitar confusiones.

4. Las estructuras en las mismas regiones anatómicas deben tener nombres armonizados.

Por otra parte, la $T H$ considerada el cuerpo oficial de nomenclatura para su uso en citología e histología humana, fue publicada por la FICAT en el año 2008, con la finalidad de reemplazar la Nomina Histologica, que se introdujo en 1977, en la cuarta edición de la Nomina Anatomica.

La TH contiene las terminologías de citología, histologías general y histología especial (es decir, anatomía microscópica). Al igual que con la TA, FICAT ha examinado y revisado los términos histológicos latinos vigentes, con la adición de una lista de términos en inglés de uso común. FICAT ha tratado de evitar cambios innecesarios y ha adoptado muchas alternativas en el uso común, con el fin de abarcar la forma variable en la que la terminología es utilizada en diferentes países. Durante el año 2003, la $T H$ fue enviada a todos los miembros de la IFAA para hacer comentarios. Así, esta terminología incorporó cambios sugeridos por estas asociaciones.

Siguiendo las recomendaciones de la FCAT (1998), FICAT (2008) y FIPAT, fueron adoptadas algunas normas para la confección, tanto de la $T A$ como de la $T H$, respectivamente. Los términos se presentan en tres columnas, con cada término latino acompañado por un término de uso actual en los países de habla inglesa. Junto a cada par existe un número de identificación único. El orden en que los términos se establecen tiende a seguir la anatomía natural a través de cada sistema. Cuando esto no es posible, los términos aparecen en orden alfabético. Sangría y estilos de 
título se utilizan para indicar la relación de un término a otro. Cuando dos o más términos alternativos están separados por ";" el primer término es preferido por el Comité. Cada terminología se completa por un índice exhaustivo de términos en latín e inglés y un índice de epónimos que identifica el número del término apropiado no eponímico. Además, se señala que sólo la lista latina de términos debe ser utilizada como base para la creación de listas de términos equivalentes en otros idiomas. Equivalentes en inglés se dan en la lista ya que este idioma se habla en muchos países, pero no es la base para la terminología en otros idiomas.

La TA comienza con una sección de términos generales y procede con las partes de cuerpo y con los términos usados para los planos, líneas y regiones. El resto de la terminología está dividida en sistemas. Cuando una estructura es sexualmente dismórfica, el mismo número es usado; sin embargo, el homólogo femenino es identificado por $q$ o el masculino $\sigma^{\top}$, cuando no es obvio que la estructura no pertenece a un sólo sexo. Muchas veces, la forma completa de un término exige partes de los nombres de las estructuras que anteceden.

Las notas al pie de página son proporcionadas para explicar la elección del término o su origen, cuando fuera adecuado. La FCAT no intenta explicar todos los términos que son de uso común. Los términos que presentan notas al pie de página son identificados con un asterisco.

La propia terminología constituye un índice, al estar dispuesta en cascada clásica descendente de lo general a lo particular, pero siguiendo la anatomía. Los títulos que son términos se les asigna un número y siguen una secuencia de mayor a menor importancia.

Por su parte, la $T H$ comienza con una sección de citología, luego continúa con la histología general de los tejidos básicos y se completa con la histología especial de órganos. Sin embargo, a pesar que la $T H$ presenta las mismas consideraciones que la $T A$ para su confección, esta última presenta algunas diferencias en cuanto a organización, denominación de términos y más de un término para una misma estructura, entre otras.

\section{Discrepancias}

Existe falta de uniformidad en el orden en que algunos términos se establecen entre una terminología y otra. Por ejemplo, al revisar el sistema digestivo se puede observar que los términos en la $T A$ se encuentran organizados de superficial a profundo, en cambio en la $T H$ la organización es de profundo a superficial (Tabla I).
También se puede observar que la misma estructura presenta nombres distintos entre una terminología y otra (Tabla II).

De acuerdo a lo señalado anteriormente, cuando existe más de uno término para identificar una estructura, el primer término es preferido por la FCAT. No obstante, al revisar ambas terminologías, se puede constatar que en numerosos casos no se sigue esta recomendación. Así por ejemplo, para una determinada estructura en la $T A$ existe un término y en la $T H$ dos términos; sin embargo, el primer término no coincide con el de la TA (Tabla III).

Al revisar ambas terminologías, se observa que además de estas discrepancias de organización de términos, denominaciones distintas para una misma estructura y dos nombres para una estructura sin coincidencia con el primer término en ambas terminologías, algunos términos están escritos en plural en la TA y singular en la TH. Algunos ejemplos se presentan en la Tabla IV.

\section{Desafíos}

De acuerdo a lo indicado por la FICAT, la terminología debe adaptarse al idioma vernáculo y su denominación debe concentrar la información y el papel descriptivo de la estructura en cuestión (Losardo et al., 2010). Sin embargo, al momento de realizar el ejercicio de escribir los términos, en este caso al español, nos encontramos con falta de precisión en la identificación de algunos términos y discrepancias entre TA y $T H$. Algunas de las mejoras a esta problemática la propone el SILAT, ya que ofrece oportunidades para revisar y discutir las distintas terminologías y así evidenciar la necesidad de modificar y armonizar los términos que presentan discrepancias. El SILAT en su estatuto enuncia actividades enfocadas a resolver este tipo de problemáticas, en el ámbito de la promoción, docencia, investigación, publicación y asesoría técnica (Losardo et al., 2015).

Por otra parte, los TICs favorecen los procesos de información y comunicación (Ávila \& Samar, 2012; Margolis, 2013) y pueden ser una buena herramienta para la actualización de TA, TH y TE. Ávila et al. (2013) señalaron la importancia de la difusión de las distintas terminologías en los diferentes sistemas, por medio del uso de internet como una vía universal y común de la comunicación en la educación médica continua y por el impacto en la práctica de la clínica médica.

Esperamos que en un futuro cercano y con más antecedentes se puedan unificar criterios, ya sea en los Comités de Terminologías de los diferentes países, como tam- 
Tabla I. Ejemplo de falta de uniformidad en algunos términos utilizados en la Terminologia Anatomica y Terminologia Histologica.

\begin{tabular}{|c|c|c|c|}
\hline \multicolumn{2}{|c|}{ TERMINOLOGIA ANATOMICA } & \multicolumn{2}{|c|}{ TERMINOLOGIA HISTOLOGICA } \\
\hline A05.4.01.001 & OESOPHAGUS & H3.04.02.1.00001 & OESOPHAGUS \\
\hline A 05.4.01.002 & Pars cervicalis; Pars colli & H3.04.02.1.00002 & Tunica тисоsa \\
\hline A 05.4.01.003 & Pars thoracica & H3.04.02.1.00003 & $\begin{array}{l}\text { Epithelium stratificatum squamosun } \\
\text { non cornificatum }\end{array}$ \\
\hline A 05.4.01.004 & $\begin{array}{l}\text { Constrictio partis thoracicae; } \\
\text { Constrictio bronchoaortica }\end{array}$ & H3.04.02.1.00004 & Cellula dendritiformis \\
\hline A 05.4.01.005 & $\begin{array}{l}\text { Constrictio phrenica; Constrictio } \\
\text { diaphragmatica }\end{array}$ & H3.04.02.1.00005 & Glandula oesophagea \\
\hline A 05.4.01.006 & Pars abdominalis & H3.04.02.1.00006 & Glandula oesophagea superior \\
\hline A 05.4.01.007 & Tunica serosa & H3.04.02.1.00007 & Glandula cardialis oesophagi \\
\hline A 05.4.01.008 & Tela subserosa & H3.04.02.1.00008 & Lamina muscularis mисоsae \\
\hline A 05.4.01.009 & Tunica adventitia & H3.04.02.1.00009 & Tela submucosa \\
\hline A 05.4.01.010 & Tunica muscularis & H3.04.02.1.00010 & Glandula submисоsa \\
\hline A 05.4.01.011 & Tendo cricooesophageus & H3.04.02.1.00011 & Mucocytus \\
\hline A 05.4.01.012 & M. bronchooesophageus & H3.04.02.1.00012 & Seromucocytus \\
\hline A 05.4.01.013 & M. pleurooesophageus & H3.04.02.1.00013 & Myoepitheliocytus \\
\hline A 05.4.01.014 & Tela submucosa & H3.04.02.1.00014 & Tunica muscularis \\
\hline A 05.4.01.015 & Tunica mucosa & H3.04.02.1.00015 & Myocytus levis internus circularis \\
\hline A 05.4.01.016 & Lamina muscularis mucosae & H3.04.02.1.00016 & $\begin{array}{l}\text { Myocytus levis externus } \\
\text { longitudinalis }\end{array}$ \\
\hline \multirow[t]{2}{*}{ A 05.4.01.017 } & Glandulae oesophageae & H3.04.02.1.00017 & $\begin{array}{l}\text { Myofibra striata visceralis non } \\
\text { cardiaca }\end{array}$ \\
\hline & & H3.04.02.1.00018 & Tunica adventitia \\
\hline
\end{tabular}

Tabla II. Ejemplo de nombres distintos utilizados para una misma estructura en la Terminologia Anatomica y Terminologia Histologica.

\begin{tabular}{|c|c|c|c|}
\hline \multicolumn{2}{|c|}{ TERMINOLOGIA ANATOMICA } & \multicolumn{2}{|c|}{ TERMINOLOGIA HISTOLOGICA } \\
\hline A05.6.01.001 & INTESTINUM TENUE & H3.04.03.0.00001 & INTESTINUM TENUE \\
\hline A 05.6.01.004 & Tunica muscularis & H3.04.03.0.00023 & Tunica muscularis \\
\hline A 05.4.01.005 & $\begin{array}{l}\text { Stratum longitudinale; Stratum } \\
\text { helicoidale longi gradus }\end{array}$ & H3.04.03.0.00024 & Myocytus levis internus circularis \\
\hline A 05.6.01.006 & $\begin{array}{l}\text { Stratum circulare; Stratum } \\
\text { helicoidale brevis gradus }\end{array}$ & H3.04.03.0.00025 & $\begin{array}{l}\text { Myocytus levis externus } \\
\text { longitudinalis }\end{array}$ \\
\hline
\end{tabular}

Tabla III. Ejemplo de más de un nombre para la misma estructura en Terminologia Histologica. No se respeta el nombre utlizado en Terminologia Anatomica, colocándose el término como segunda opción.

\begin{tabular}{lllc}
\hline \multicolumn{2}{c}{ TERMINOLOGIA ANATOMICA } & \multicolumn{2}{c}{ TERMINOLOGIA HISTOLOGICA } \\
\hline A05.7.05.001 & CANALIS ANALIS & H3.04.04.1.00001 & CANALIS ANALIS \\
A05.7.05.009 & Linea pectinata & H3.04.04.1.00011 & Linea dentata; Linea pectinata \\
\hline
\end{tabular}

Tabla IV. Ejemplo que muestra la falta de concordancia en el uso del plural y singular para un mismo término entre la Terminologia Anatomica y Terminologia Histologica.

\begin{tabular}{llll|}
\hline \multicolumn{2}{c}{ TERMINOLOGIA ANATOMICA } & \multicolumn{2}{c}{ TERMINOLOGIA HISTOLOGICA } \\
\hline A05.1.04.001 & LINGUA & H3.04.01.0.03001 & LINGUA \\
A05.1.04.014 & Papillae filiformes & H3.04.01.0.03004 & Papilla filiformis \\
A05.2.01.001 & FAUCES & H3.04.01.0.04001 & FAUCES \\
A05.2.01.014 & Fossulae tonsillea & H3.04.01.0.04004 & Fossula tonsillae \\
\hline A05.3.01.001 & PHARYNX & H3.04.01.1.00001 & PHARYNX \\
\hline A05.3.01.030 & Glandulae pharyngeales & H3.04.01.1.00004 & Glandula pharyngea \\
\hline
\end{tabular}


bién en los SILAT y también que estas recomendaciones puedan ser finalmente consideradas por los expertos que conforman el FIPAT, en beneficio de la comunidad científi- ca internacional y especialmente para aquellos que se dedican a la enseñanza de las diferentes áreas de las ciencias morfológicas del área de la medicina.

VÁSQUEZ, B. \& DEL SOL, M. Terminologia Anatomica and Terminologia Histologica. A meeting point between morphologists. Int. J. Morphol., 34(4):1585-1590, 2015.

SUMMARY: The correct use of morphological terminology is extremely important for scientific communication. These terminologies should be clear and precise and consistent with each other. The need to unify criteria has been reflected in the performance of the Iberian Latin American Symposium of Terminology (SILAT), initiated in 2009 by the Pan American Association of Anatomy. The priority is to unify and update morphological terms and also pose challenges and propose solutions to improve the different terminologies. The objective was to coordinate a review of some Latin terms common to Terminologia Anatomica (TA) and Terminologia Histologica (TH), in order, by way of example compare and identify some terms that generate discrepancy, and therefore confusion for those who use it. Although both terminologies present the same considerations for their makeup, there are some differences in organization, denomination of terms and more than one single term for the same structure. Furthermore, the same structure may have different names between one terminology and another. Many terms are written in the plural and singular in TA and TH. SILAT proposes some improvements for these issues by providing opportunities to review and discuss the different terminologies and thus demonstrate the need to modify and harmonize terms that present discrepancies. In short, we are hopeful that with this initial information, arguments can be made to further uniform criteria that may be considered by the experts that make up the Federative International Programme for Anatomical Terminology. Additionally, and as noted this would allow the development of dialogue with members of the International Federation of Associations of Anatomists, and continue to improve scientific communication between the various actors of the morphological sciences.

\section{KEY WORDS: Morphological terminologies; Terminologia Anatomica; Terminologia Histologica.}

\section{REFERENCIAS BIBLIOGRÁFICAS}

Ávila, R. E \& Samar, M. E. Medicina Virtual: Recursos digitales en Educación Médica. Experiencias en docencia, investigación y producción. Editorial Académica Española (EAE), LAP LAMBERT Academic Publishing GmbH \& Co. KG. Saarbrücken, 2012.

Ávila, R.; Samar, M.; Sugand, K.; Metcalfe, D.; Evans, J. \& Abrahams, P. The first South American free online virtual morphology laboratory: Creating history. Creative Education, 4:6-17, 2013.

Cruz, G. R.; Rodríguez, T. A.; Prates, J. C.; Losardo, J. R. \& Barbato, V. N. Simposios Ibero-Latinoamericanos de terminología. Anatómica, histología y embriología. Int. J. Morphol., 28(1):333-6, 2010.

del Sol, M. \& Vásquez, B. Median cephalic and median basilic veins. Why their exclusion from Terminologia Anatomica? Int. J. Morphol., 32(2):721-4, 2014.

Duque Parra, J. E.; Barco, R. J. \& Duque, Q. N. Depurando la Terminologia Anatomica: apertura y foramen, dos similitudes y redundancia terminológica. Int. J. Morphol., 30(4):1497-9, 2012.

Duque, J. E. \& Ríos, J. Umbilical-spinous line: a morphological term that should be included in the anatomical terminology. Colomb. Med. (Cali), 44(3):202-4, 2013.
Federative Committee on Anatomical Terminology (FCAT). Terminologia Anatomica: International Anatomical Terminology. Stuttgart, Georg Thieme Verlag, 1998.

Federative International Committee on Anatomical Terminology (FICAT). Terminologia Histologica: International Terms for Human Cytology and Histology. Philadelphia, Wolters Kluwer/ Lippincott Williams \& Wilkins, 2008.

Fuentes, R.; Ottone, N.; Cantín, M. \& Bucchi, C. Analysis of terms used in scientific literature referred to extracapsular ligaments of the temporomandibular joint. Part II:Ligamentum stylomandibulare and Raphe pterymandibulare. Int. J. Morphol., 32(4):1289-95, 2014.

Latarjet, M. \& Ruiz, L. A. Anatomía Humana. $4^{\mathrm{a}}$ ed. Panamericana, Madrid, 2004.

Losardo, R. J.; Cruz Gutiérrez, R.; Rodríguez Torres, A.; Prates, J. C. \& Valverde Barbato de Prates, N. Iberia-Latin-American Symposia of Morphological Terminology (SILAT). First two years and statute. Int. J. Morphol., 28(4):1323-6, 2010.

Losardo, R. J.; Prates, N. E. V. B.; Arteaga-Martínez, M.; Cabral, R. H. \& García-Peláez, M. I. Terminología morfológica internacional: Algo más que anatomía, histología y embriología. Int. J. Morphol., 33(1):400-7, 2015. 
Margolis, A. Tendencias en educación médica continua a distancia. Inv. Ed. Med., 2(1):50-4, 2013.

Mejías S. R. El latín como lengua de la terminología anatómica: Algunas observaciones sobre su rol. Rev. Arg. Anat. Online, $3(2): 33-42,2012$.

Parra. G. L.; Ibarra, R. V.; González, R. J. \& García, H. A. El reconocimiento de conceptos básico-clínicos bajo la terminología anatómica internacional hacia la formación médica. Inv. $E d$. Med., 4(15):139-44, 2015.

Roa, I. \& del Sol, M. Clarificación de la Terminologia Embryologica: conducto de Gartner. Int. J. Med. Sug. Sci., 2(2):481-6, 2015.

Vásquez, B. \& del Sol, M. The Terminologia Histologica in the medical sciences. Int. J. Morphol., 32(1):375-80, 2014.

\section{Dirección para Correspondencia: Dra. Bélgica Vásquez Pastene Universidad de Tarapacá \\ Arica - CHILE}

Email: bvasquezp@uta.cl

Recibido : 25-09-2015.

Aceptado: 18-11-2015. 